Horizons philosophiques

\title{
La science contemporaine connaît-elle vraiment le monde?
}

\section{Gilles Lane}

Volume 2, numéro 2, printemps 1992

Philosophie et sciences : du concept au réel

URI : https://id.erudit.org/iderudit/800902ar

DOI : https://doi.org/10.7202/800902ar

Aller au sommaire du numéro

\section{Éditeur(s)}

Collège Édouard-Montpetit

\section{ISSN}

1181-9227 (imprimé)

1920-2954 (numérique)

Découvrir la revue

\section{Citer cet article}

Lane, G. (1992). La science contemporaine connaît-elle vraiment le monde?

Horizons philosophiques, 2(2), 185-203. https://doi.org/10.7202/800902ar d'utilisation que vous pouvez consulter en ligne.

https://apropos.erudit.org/fr/usagers/politique-dutilisation/ 


\section{La science contemporaine connaît-elle vraiment le monde?}

Quels sont les gens qui posent cette question? Et pourquoi la posent-ils? Parmi eux, il y a ceux qui ont des doutes à ce propos et qui voudraient bien savoir quoi penser. Mais il y a surtout ceux qui en connaissent déjà la réponse, et pour qui cette réponse est «non». D'autres aussi disent non, mais il s'agit d'un "non" qui veut surtout dire "pas vraiment», ou "pas aussi bien» ou «aussi profondément» que je voudrais le connaître, ce monde. Plusieurs de ces derniers s'opposent d'autant plus à reconnaître le caractère proprement cognitif de la science que de nombreux scientifiques, philosophes des sciences ou citoyens profanes, ne jurent que par la science et méprisent les prétentions naïves de toute démarche non scientifique, à une véritable connaissance de quoi que ce soit. Ces opposants concèdent parfois que la science contemporaine permet sans doute de connaître une foule de choses, mais non pas de les comprendre - ce qui, selon eux, serait vraiment les connaître.

II fut un temps où les scientifiques - les chercheurs sérieux et ouverts, comme aussi les simples opportunistes - étaient la plus grande autorité en matière de connaissances, étant donné leurs succès si impressionnants dans les sciences expérimentales et techniques, tout comme dans les sciences théoriques (ou «pures»). À partir du 
XVIle siècle, peut-être davantage avec Newton, le progrès et l'apport des mathématiques se sont mis à favoriser la formulation de lois de la nature de même que l'application de celles-ci aux résultats d'observations et d'expériences de plus en plus nombreuses et précises. On obtint, par ces lois, une connaissance toujours plus détaillée, plus favorable que jamais à de nouvelles recherches et découvertes, qui permettait une foule d'applications pratiques fort appréciables. Encore aujourd'hui, de nouvelles lois scientifiques sont sans cesse découvertes dans de nombreux domaines, leur utilité pratique n'ayant pas à être démontrée.

\section{Les sciences théoriques}

II faudrait d'abord noter que les plus grands avantages des lois scientifiques, tant du point de vue utilitaire que du point de vue connaissance du monde, sont fournis par la composante technique des sciences contemporaines. Les sciences techniques sont celles qui cherchent non seulement à exploiter les lois déjà découvertes, mais aussi à en trouver de nouvelles qui permettraient d'utiliser les objets et les forces de la nature pour un profit que l'on a déjà en vue ou que l'on pourrait découvrir plus tard. Les sciences techniques sont d'abord et avant tout la recherche d'un savoir-faire, sinon uniquement cela.

Quant aux sciences théoriques, elles visent en premier lieu la connaissance elle-même. On peut même dire qu'elles n'ont aucun souci des applications pratiques, ni aucun intérêt pour la transformation du réel en vue d'avantages que les chercheurs eux-mêmes, ou d'autres personnes, pourraient en tirer. Si jamais quelque profit pouvait être obtenu par cette connaissance, les théoriciens s'en réjouiraient sans doute - même si plusieurs d'entre eux se méfieraient de cela, comme d'un danger pour l'«objectivité" de la recherche "pure» -, mais il n'en demeure pas moins que leur intérêt concerne d'emblée la connaissance elle-même, ou soi-disant pour elle-même. (On peut 
penser qu'ils en tirent tout de même un certain profit, mais qui ne serait pas celui d'une maîtrise ou d'une future jouissance du monde lui-même.) Voulant connaître l'univers, les théoriciens cherchent donc à l'expliquer ou à le comprendre.

Les sciences théoriques ne peuvent se contenter, en effet, d'observer, d'explorer puis de décrire l'univers. Comme les sciences pratiques et techniques, elles cherchent à découvrir et à formuler nettement les diverses lois de ses "comportements", mais seulement pour en obtenir une meilleure connaissance. Plusieurs d'entre eux ont exprimé leur visée principale : connaître l'univers en découvrant surtout pourquoi il est comme il est et non pas autrement. La connaissance scientifique n'est donc pas une simple constatation ou contemplation du donné, mais une explication et, du même coup, une "compréhension" de ce donné. On peut noter, ici, que les sciences théoriques ont en cela une visée semblable à celles des diverses philosophies, y compris celles qui cherchent pourtant un autre genre de connaissance que la connaissance typiquement scientifique. On pourra constater, plus loin, que plusieurs philosophes cherchent en fait à connaître pour mieux comprendre, plutôt qu'à comprendre pour mieux connaître, même si les scientifiques d'aujourd'hui sont de plus en plus nombreux à partager cette visée plus "philosophique».

II y a deux principaux types d'explication dans les sciences théoriques : l'explication causale et l'explication formelle, étant aujourd'hui la plus recherchée ou la moins suspecte. On a une explication causale du donné observable - ou des phénomènes - lorsqu'on a réussi à découvrir (ou à imaginer) une cause qui ferait que ces phénomènes sont comme ils sont, plutôt qu'autrement. II ne faudrait pas oublier, cependant, que c'est le donné observable qu'il s'agit toujours de connaître - et donc d'expliquer ou de comprendre - et non pas ses causes ellesmêmes qui ne serviraient qu'à fournir une meilleure connaissance du seul donné. C'est d'ailleurs la raison pour 
laquelle de nombreux théoriciens d'aujourd'hui estiment qu'afin de mieux connaître les phénomènes, il n'est pas nécessaire que leurs causes "explicatives" existent, ni qu'elles soient observées, ni même en principe observables.

Selon le modèle "causal» de l'explication scientifique, le chercheur imagine donc des entités dites "théoriques" - par exemple, des particules subatomiques ou des forces nucléaires - auxquelles il attribue des comportements réglés par des lois déjà connues ou simplement postulées, le but étant de pouvoir ensuite considérer les phénomènes comme découlant réellement, ou au moins logiquement, de ce qu'il a ainsi imaginé ou posé (et qui constitue une théorie explicative). Le chercheur "causaliste" a la conviction de mieux connaître le donné observable - c'est-à-dire de l'avoir expliqué et vraiment compris - lorsqu'il est parvenu à le percevoir comme découlant, de quelque façon, de la théorie qu'il a conçue et structurée de manière à ce que cette déduction soit justement possible. De plus, il vérifie ce qu'on appelle la "validité" de cette théorie - et de la connaissance qu'elle permet d'obtenir - en cherchant à voir si elle peut tenir le coup sous l'épreuve de nouvelles observations et expériences "scientifiques" et en modifiant, au besoin, ses entités et structures initiales pour qu'elle puisse résister à cette épreuve du réel lui-même. (Depuis quelques années, les créateurs des théories notamment en physique - reconnaissent que leurs théories ne peuvent jamais être parfaitement ni définitivement vérifiées, et qu'ils doivent donc se contenter de celle qui leur semble être, ou qu'ils sentent être, la moins vulnérable ou la plus prometteuse.)

Ces adeptes de l'explication causale se partagent en deux groupes d'inégale importance : les "réalistes" (moins nombreux, mais en croissance depuis qu'ils se sont mis à nuancer leur conception du "réel») et les "formalistes" plus ou moins "purs». Ces deux étiquettes veulent souligner le fait que les premiers attribuent encore un caractère 
de réalité, d'existence et de causalité aux entités théoriques et à leurs comportements, alors que les seconds leur refusent ce caractère. (On verra, plus loin, que la plupart de ces derniers ont préféré abandonner complètement le concept même de causalité dans les sciences théoriques pour devenir des formalistes «purs».)

II importe de noter tout de suite que les théoriciens réalistes prêtent le flanc à une objection sérieuse en ce qui concerne leur connaissance du monde observable. En effet, comme tous les théoriciens scientifiques, leur souci principal, au départ, est de découvrir pourquoi (ou comment il se fait que) les phénomènes sont comme ils sont et non pas autrement. Ils ont la conviction que telle ou telle théorie "explicative» leur apporte déjà, comme simple théorie, une certaine réponse à cette question, et par conséquent, une certaine connaissance du monde observable. Or, même si une théorie devenait un jour si parfaite qu'on ne puisse plus la trouver en contradiction avec de nouvelles observations et expériences, on pourrait sans doute se demander : "Comment ces "réalistes" pourraient-ils croire qu'ils ont obtenu une véritable réponse à leur question principale alors qu'ils ne sauraient toujours pas pourquoi, en fait, les entités et les comportements théoriques - qui sont censés causer les particularités du donné observable - sont eux-mêmes comme ils sont et non pas autrement?»

$\mathrm{Au}$ lieu de rechercher des causes réelles, mais de moins en moins observables, qui contraindraient les phénomènes à être comme ils sont (ou d'imaginer des causes fictives pouvant exercer cette même contrainte), les théoriciens «formalistes» s'efforcent d'expliquer les phénomènes en cherchant à les faire entrer dans des structures appropriées. À la question: «Pourquoi les planètes tournentelles autour du soleil?", les formalistes répondraient : "Parce qu'elles ont à former des ellipses autour du soleil." (À l'époque de Ptolémée, ils auraient sans doute répondu qu'il n'existe pas de "sphères" célestes qui entraînent les 
planètes autour de la terre, mais que celles-ci ont à tourner autour de la terre, un point c'est tout.)

Plutôt que le causalisme, un grand nombre de formalistes contemporains ont donc préféré un structuralisme purement légaliste. $\mathrm{Si}$, par exemple, on aperçoit quelque part dans la nature des rochers qui sont tout simplement là où ils sont, on pourra surmonter son propre étonnement - ou sa propre ignorance - en observant tout à coup que ces rochers sont précisément là où ils sont parce qu'ils ont à former ensemble, disons, un cercle (ou un losange, ou telle ou telle figure plus complexe). La théorie de la relativité ne fait rien d'autre que cela, en principe. Elle a prévu avec raison, en effet, qu'elle n'aurait plus besoin de faire intervenir des causes - ou toute autre uforce" trop mystérieuse ou trop «métaphysique» — si elle parvenait à concevoir au moyen des mathématiques (le calcul «tensoriel» en l'occurrence), une structure générale de l'univers, c'est-à-dire une "géométrie» plus abstraite qui imposerait "légalement" aux corps de la nature les comportements qu'ils devraient nécessairement avoir lorsque laissés à euxmêmes.

Les formalistes ont sans doute l'avantage, par rapport aux causalistes plus intéressés à l' «étoffe" des êtres, d'expliquer les structures observables du monde au moyen de lois plus simples. Pour Einstein, par exemple, on sait que plus notre conception du monde devient simple, plus elle s'approche de "la réalité". Il est évident, en tout cas, que l'unification des données observables, au sein d'une structure aussi précise et simple que possible, est très utile. Elle permet non seulement de réduire le nombre de nos questions et problèmes, mais d'acquérir en même temps un point de vue sur le monde qui en rende la multiplicité et la variété moins déroutantes ou moins mystérieuses. On peut toutefois se demander s'il s'agit là d'une connaissance théorique ou plutôt d'une connaissance pratique dont l'utilité ne serait que purement psychologique.

II faut se le rappeler : les théoriciens scientifiques 
cherchent uniquement à augmenter ou à améliorer leurs connaissances des réalités observables, et cela, au moyen de théories particulières qui se veulent typiquement "scientifiques". La plupart d'entre eux croient - comme beaucoup de leurs adeptes profanes - que ce moyen est le seul à pouvoir livrer une véritable connaissance de quoi que ce soit, tout au moins la meilleure ou la plus fiable des connaissances possibles. Voyons donc de plus près en quoi consiste, au juste, ce type de connaissance, et en quoi la théorie scientifique permettrait d'augmenter ou d'améliorer nos connaissances elles-mêmes.

\section{La «connaissance» théorique}

Qu'est-ce que vient ajouter, aux connaissances que nous acquérons déjà par l'observation et l'exploration du monde et de ses lois observables, le fait qu'on réussisse à déduire ce monde (et ces lois) d'un certain nombre d'entités et de comportements purement théoriques? Ou le fait qu'on réussisse à concevoir leurs structures comme faisant partie de structures plus vastes ou plus générales et unifiantes? Que sait-on de plus sur le monde lui-même, si ce n'est qu'il est tel qu'on puisse le déduire comme on l'a fait ou qu'on puisse l'intégrer dans ces structures particulières? Qu'est-ce qu'on aurait appris de plus, à propos de ce monde, lorsqu'on aurait pu le déduire d'entités et de comportements théoriques encore plus simples ou l'intégrer dans les structures les plus simples possibles? II semble qu'il n'y aurait pas beaucoup de différence, en effet, entre croire qu'une théorie scientifique a augmenté nos connaissances, et croire que l'on a augmenté ou amélioré ses propres connaissances de ces deux oranges-ci par exemple, du seul fait que l'on ait réussi à les insérer toutes les deux dans la plus petite boîte que l'on aurait pu dénicher...

S'il y a réellement une différence entre ces deux cas, concernant l'augmentation ou l'amélioration de nos connaissances, il faudrait bien pourtant la découvrir ou la montrer.Cependant s'il n'y en a pas ou si l'on ne la voit pas, 
il serait alors difficile de penser autre chose : les théoriciens scientifiques s'intéressent à l'élaboration de leur type particulier de théories pour d'autres raisons, en fait, que l'augmentation ou l'amélioration de leurs connaissances du monde lui-même. Ce pourrait être, par exemple, pour le plus grand plaisir que ce travail leur apporte ou parce que toute autre façon de chercher à mieux connaître la réalité les rendrait beaucoup trop insécures ou parce qu'ils détestent toute autre méthode de recherche, ou les implications que les résultats de ces autres méthodes pourraient contenir ou parce que, tout simplement, ils ont éprouvé une grande admiration, tout jeunes, à l'endroit des théoriciens scientifiques et n'ont pu s'empêcher de croire que ces savants possédaient la meilleure connaissance possible de quoi que ce soit.

II serait sans doute injuste de penser que tous les théoriciens ont des raisons ou des motifs de ce genre, mais il serait toutefois naiff de penser qu'aucun d'eux n'est principalement motivé par de semblables désirs ou réticences. II est donc difficile d'éliminer de telles possibilités, surtout lorsqu'on ne voit pas très bien comment notre connaissance de telle ou telle chose ait pu être vraiment accrue, ou améliorée, du seul fait que l'on ait réussi à fabriquer les «tenailles» conceptuelles avec lesquelles on pouvait saisir les choses le plus simplement ou le plus aisément possible. Quoi qu'il en soit des motifs des théoriciens, il n'en reste pas moins que leur science n'est qu'un jeu conceptuel qui n'avance en rien notre connaissance du monde. En effet, les sciences techniques s'intéressent uniquement aux transformations et exploitations utiles du monde lui-même, elles recherchent surtout les lois les plus diverses et les plus particulières de ce monde - plutôt que des théories "unitaires», ou soi-disant "explicatives» -, et contribuent bien davantage, elles, à la connaissance (pratique) de notre monde. 


\section{Résumé}

Selon les sciences théoriques d'aujourd'hui, les choses de l'univers, et l'univers lui-même, sont comme ils sont et se comportent comme ils se comportent (et non pas autrement) parce que, à cause de $X$, ils ont à être comme ils sont, et se comporter comme ils se comportent. Quant à X lui-même, il s'agit ou bien d'un ensemble de choses et de forces faisant partie de l'univers, ou bien d'un ensemble de lois que les choses de l'univers, et leurs comportements, ne peuvent éviter de suivre. En fait, l'idéal des sciences théoriques serait complètement atteint, dans les deux cas, si l'on découvrait un ensemble $X$ qui permettrait de savoir pourquoi, à chaque instant, les différentes choses de l'univers sont comme elles sont, et se comportent comme elles se comportent. L'ensemble X permettrait donc de mieux connaître les choses de l'univers, et leurs comportements.

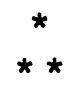

Comment se fait-il, cependant, que les chercheurs théoriques ne semblent pas intéressés à savoir pourquoi les choses et les forces de l'ensemble $X$ sont comme elles sont ou se comportent comme elles se comportent (et non pas autrement)? Ou pourquoi les lois ou structures de l'ensemble $X$ sont justement celles-là, plutôt que d'autres? Comment peuvent-ils s'arrêter là, alors qu'ils semblent pourtant vouloir connaître le pourquoi des choses et, en principe, le pourquoi de quoi que ce soit? Serait-ce seulement parce qu'ils n'ont pas encore réussi à découvrir l'ensemble $X$ qu'ils cherchent depuis si longtemps? Mais ne voient-ils pas qu'en renonçant à découvrir le pourquoi de l'ensemble $X$ lui-même (quel qu'il soit!), ils renonceraient à une véritable connaissance de l'univers ou du moins à une connaissance du monde qui serait vraiment meilleure? Lorsqu'un chercheur veut connaître le pourquoi des choses 
et de leurs comportements - et non pas seulement découvrir de nouvelles choses, de nouveaux comportements ou les lois particulières qu'ils auraient à suivre -, il semble qu'il ne puisse s'arrêter en cours de route, à moins, bien sûr, que son désir principal n'ait jamais été, celui d'une véritable connaissance du monde.

Il est évident qu'on ne saurait imposer à qui que ce soit de vouloir rechercher la meilleure connaissance possible de l'univers, comme on ne saurait penser, non plus, que la vie humaine la plus valable est celle qui serait consacrée à la recherche de la meilleure des connaissances. On peut toutefois avoir le goût d'attirer l'attention sur certaines méprises de notre culture d'aujourd'hui, surtout lorsqu'elles concernent les sciences théoriques dont la réputation, en matière de connaissance, est due à peu près uniquement aux succès des sciences purement techniques.

\section{La connaissance "philosophique»}

La plupart des philosophes contemporains cherchent aussi la réponse à la question : «Pourquoi les êtres sont-ils comme ils sont, ou se comportent-ils comme ils se comportent, et non pas autrement? " Plusieurs d'entre eux croient, comme les théoriciens scientifiques, que cette réponse devrait être obtenue au moyen d'une explication du monde et de ses objets, c'est-à-dire lorsqu'on réussirait à les voir comme découlant d'autres objets mondains (ou purement conceptuels) ou comme ayant à faire partie de structures plus ou moins abstraites. En d'autres termes, ce que ces philosophes et ces théoriciens scientifiques voudraient principalement faire, au moyen de ce type d'explication, c'est sortir les réalités - de même que leurs propriétés et leurs comportements - de l'arbitraire.

On peut toutefois remarquer que le désir de connaissance chez les philosophes d'hier et d'aujourd'hui - et même chez de nombreux profanes - possède un trait assez caractéristique. II s'agit en effet d'un désir de con- 
naissance qui ne leur permet pas de s'arrêter à cette seule explication du monde. Pour eux, toute explication nouvellement découverte contient encore de l'arbitraire, et relance donc indéfiniment leur désir d'une meilleure connaissance des réalités. À cause de cela, on comprend que l'amélioration cognitive qui est désirée et recherchée ne peut pas être celle que fournirait ce type d'explication des réalités déjà connues, mais doit être plutôt celle que leur apporterait ce qu'on peut appeler une compréhension de celles-ci (même lorsque déjà expliquées).

Alors que les chercheurs scientifiques présupposent qu'il devrait y avoir moyen d'expliquer les choses et les faits qui sont tout simplement là, devant eux, les philosophes présupposent, de leur côté, qu'il devrait y avoir moyen de comprendre ces mêmes choses et ces mêmes faits, toujours si arbitraires. Les philosophes ne se demandent pas pourquoi les êtres sont comme ils sont plutôt qu'autrement, ils ne cherchent pas ce qui ferait qu'ils sont ainsi plutôt qu'autrement ou pourquoi ils ont à être ainsi plutôt qu'autrement. Ils se demandent surtout quoi penser du fait que les êtres sont comme ils sont, ou comment réagir à ce fait arbitraire. (lls ne pourraient que se demander encore la même chose, en effet, s'ils avaient découvert ce qui ferait que les réalités sont comme elles sont, puisqu'ils se trouveraient devant des faits nouveaux, mais tout aussi arbitraires...) Au lieu de se contenter d'une explication des faits par d'autres faits (réels ou purement formels), ces philosophes recherchent donc, en plus, ce qu'on pourrait appeler le "sens» des faits, un sens qui, en sortant ces faits de leur pur arbitraire, leur permettrait enfin de les comprendre.

Que manque-t-il donc aux philosophes typiques - et qui ne semble pas manquer du tout aux scientifiques théoriques - pour que leur désir de vraiment connaître les réalités de l'univers demeure toujours insatisfait, même lorsqu'ils réussissent à formuler des théories «philosophiques» leur permettant d'expliquer, eux aussi, (de façon 
sans doute moins précise mais plus substantielle) pourquoi les choses sont comme elles sont et non pas autrement?

Quel est le sens qu'ils désirent découvrir et dont l'absence les empêche de penser qu'ils auraient enfin surmonté cet arbitraire de l'univers (et de leurs propres théories)? II est pourtant évident que les êtres et leurs comportements, de même que leur totalité plus ou moins constante ou variable, conserveront toujours, en eux-mêmes, un caractère inévitablement arbitraire. Car tout cela - y compris surtout la totalité de l'univers - sera toujours, à chaque instant, comme ceci et non pas autrement. Par conséquent, si jamais le désir de connaissance des philosophes les plus typiques devait pouvoir être satisfait, il faudrait nécessairement que ce soit par la découverte d'un sens atteint à travers, ou dans une connaissance (ou une explication) de faits toujours arbitraires.

Si ce genre de découverte se produisait - on verra que le cas est tout de même assez fréquent -, l'arbitraire ne disparaîtrait pas pour autant, mais il ne serait plus un problème accaparant puisque le chercheur connaîtrait le sens qu'il recherchait et le comprendrait. Comme simples "supports" du sens, il importe peu, en effet, que les choses de l'univers et leurs comportements soient comme ils sont et non pas autrement. Ce n'est sans doute pas le lieu, ici, d'exposer en long et en large ce que pourrait être ce sens, c'est-à-dire l'objet de la sorte de connaissance que désirent les philosophes typiques (de même que bien des gens peu instruits!). Mais l'exemple suivant pourra au moins, illustrer la possibilité que la connaissance d'un monde factuel et nécessairement arbitraire permette malgré tout la découverte et la compréhension d'un «sens» désiré.

\section{La connaissance "compréhensive»}

Avant de présenter cet exemple, je voudrais pourtant rappeler que "parler à quelqu'un" consiste, le plus souvent, à produire des sons, des gestes ou des choses, mais de manière à ce que l'autre, en percevant tout cela, pen- 
sera à ce à quoi on voudrait qu'il pense (et non pas à autre chose). On tâche alors de s'assurer par exemple, qu'en entendant le son "chien", l'autre pensera à un élément bien particulier d'une arme à feu et non pas à l'animal que le même son peut aussi évoquer. (Bien que "motivés", les sons d'une langue sont arbitraires...) On tâche encore, au besoin, d'attirer l'attention de l'autre sur le fait qu'on veut lui dire quelque chose, et même de susciter assez d'intérêt ou de curiosité, pour qu'il désire découvrir celamême à quoi l'on voudrait qu'il pense (au lieu de laisser venir n'importe quelle pensée).

Venons-en maintenant à l'exemple annoncé. François $(F)$ vient de dire quelque chose à Mireille $(M)$. Celle-ci est donc devant un fait - un certain nombre de sons - et voit tout de suite ce fait comme ayant été produit par $F$. À cause du regard de ce dernier, elle pense aussi que $F$ a produit les sons en question non seulement pour qu'elle les entende, mais aussi (et surtout) pour lui dire quelque chose au moyen de ces sons. En d'autres termes, $F$ a réussi à faire penser à $M$ qu'il voulait qu'elle pense en ce moment à une certaine chose ou qu'elle pense, au moins, qu'il y avait quelque chose à comprendre - c'est-à-dire un «sens» - «dans» ou "à travers" les sons qu'il avait produits. Considérons maintenant le cas où $M$ a tout de suite la conviction que $F$ veut lui dire ou lui faire penser à ceci à propos de cela. Par le fait même, elle sait pourquoi les sons qu'elle a perçus étaient comme ils étaient, et non pas autrement : c'est parce que $\mathrm{F}$ les avaient produits tels qu'elle puisse penser à cela-même à quoi il voulait justement qu'elle pense. Considérons le cas, aussi, où $M$ se demande ensuite pourquoi $F$ voulait lui dire ce qu'il lui a dit (et non pas autre chose). Elle fait quelques hypothèses : c'était pour l'avertir d'un danger; ou pour la renseigner sur telle ou telle chose; ou simplement pour engager la conversation. Si elle croit que c'était en fait pour la renseigner sur une certaine chose, elle pourrait encore se demander 
pourquoi il aurait voulu lui parler de cela au lieu d'autre chose; ou à quoi il voulait en venir, en fin de compte.

Si $M$ désirait découvrir pourquoi $F$ voulait lui parler de A au lieu de B - et s'il avait voulu parler de B, alors pourquoi de $B$ au lieu de $C$, ou de $A-$, elle aurait sans doute procédé comme le font les chercheurs théoriques, lorsqu'ils sont en quête d'une explication des faits observables. Elle serait passée par une série d'hypothèses $F$ désirait lui fournir un renseignement appréciable; ou de converser avec une femme charmante; ou de lui faire montre de ses connaissances - , mais pour se demander ensuite pourquoi $\mathrm{F}$ a préféré tel plaisir à tel autre ou pourquoi ses désirs (ou leurs causes) étaient comme ils étaient ou alors pourquoi les structures auxquelles ses désirs avaient à obéir étaient comme elles étaient et non pas autrement. Et ainsi de suite. Tout comme les chercheurs théoriques, $M$ n'aurait pas pu se débarrasser de l'arbitraire...

Mais si, au lieu de penser que $F$ était déterminé à vouloir faire ce qu'il a voulu faire, $M$ a pensé qu'il avait choisi lui-même, donc librement, de lui dire ce qu'il lui a dit, et cela, pour lui donner un renseignement qu'elle pourrait être contente d'obtenir, alors $M$ aurait pu se demander pourquoi $F$ avait choisi de lui donner ce renseignement. Et sa question n'aurait pas été, cette fois, de savoir pourquoi $F$ avait voulu la renseigner au lieu d'avoir voulu faire autre chose - n'étant pas déterminé, $F$ pouvait en effet choisir entre une foule de choses -, mais plutôt celle de savoir à quoi $F$ voulait en venir en plus de lui faire penser qu'il voulait la renseigner et lui faire plaisir. En d'autres termes, $M$ aurait pu désirer savoir ce que $F$ voulait encore faire en lui donnant ce renseignement; ou quel était le sens que $F$ ait voulu le lui donner. II se peut que $M$ ait eu tout a coup la conviction que $F$ voulait lui faire penser au fait qu'il s'intéressait à elle, non seulement à ses qualités charmantes et à ses capacités impressionnantes, mais aussi, et surtout, à elle-même. Elle aurait alors compris le sens que $F$ ait choisi librement de lui donner ce renseignement - sans 
doute aidée, en cela, par la perception d'une certaine attitude de $F$ de même que par le désir qu'elle éprouvait peutêtre déjà, plus ou moins consciemment, de recevoir un semblable intérêt (soit de $F$, soit des personnes en général).

Le fait que $F$ ait choisi d'offrir ce renseignement à $M$, mais comme moyen de lui faire comprendre qu'il s'intéressait lui-même à elle, est encore arbitraire, puisque $F$ aurait certainement pu employer d'autres moyens pour lui faire comprendre la même chose. $F$ a tout simplement profité de l'idée qu'il a eue, tout à coup, d'atteindre son but en utilisant ce moyen arbitraire (parmi d'autres moyens possibles, mais tout aussi arbitraires). Et cet arbitraire demeurera toujours, puisqu'il serait toujours impossible de suivre jusqu'au bout la chaîne des causes réelles (ou purement conceptuelles) qui se seraient déterminées les unes les autres, successivement, à déterminer $\mathrm{F}$ à avoir cette idée; ou de reconstituer la loi unique à laquelle l'émergence de cette idée aurait eu à obéir.

De plus, il est clair que $F$ aurait pu procéder d'une manière beaucoup plus directe, en disant à $M$, par exemple : “Je m'intéresse à toil», de sorte que $M$ n'aurait pas eu à deviner ... Mais $\mathrm{F}$ savait peut-être, par expérience, que, de toute façon, $M$ aurait pu éprouver le besoin de vérifier sa compréhension instantanée de ce qu'il lui disait - c'est-à-dire la réalité de cet intérêt de F à son égard et qu'il lui faciliterait sans doute la tâche en lui donnant à comprendre (comme ce fut le cas) un fait observable, c'està-dire cette offre d'un renseignement appréciable plutôt qu'une affirmation aussi générale que «Je m'intéresse à toi!»

La question du sens que pourrait avoir une personne de choisir librement de s'intéresser à d'autres personnes est une question qui ne se pose pas, puisqu'on en connaît déjà la réponse. En effet, pourquoi ou pour faire quoi, choisit-on (librement) de s'intéresser à des personnes (ou d'avoir toute autre attitude "positive" à leur égard)? La 
réponse à cette question ne peut être que celle-ci : pour s'intéresser ou pour en arriver à s'intéresser soi-même à ces personnes. Car, choisir de s'intéresser à des personnes, ce n'est pas identiquement la même chose que de s'intéresser ou que d'être intéressé, à ces personnes. II s'agit cependant du seul cas où choisir de faire une chose est nécessairement suivi du fait de faire cette chose. (Au contraire, choisir d'aller prendre l'air, de manger quelque chose, de parler à d'autres, etc., n'est pas inévitablement suivi de l'idée de prendre effectivement de l'air, de manger, etc.) La personne qui choisit librement de s'intéresser ellemême, ou d'elle-même, à d'autres personnes, connaît donc le sens de ce fait : elle produit et utilise en effet ce choix pour s'intéresser à ces personnes.

Tiré de nos expériences du langage, cet exemple a pu montrer comment il était possible, en ce qui concerne certains faits pourtant arbitraires, de vraiment satisfaire le désir de les connaître, c'est-à-dire de répondre à la question : "Pourquoi ces faits sont-ils comme ils sont et non pas autrement?" Comme on l'a vu, il s'agissait de découvrir, "à travers" ou "dans" ces faits, le sens particulier qui était actuellement à comprendre. Mais parce que tiré de l'usage que nous faisons du langage, cet exemple semble impliquer que nul ne saurait vraiment satisfaire son propre désir d'une connaissance théorique - ce désir de connaître soi-disant seulement pour connaître - s'il avait décidé de limiter l'objet de la connaissance désirée à n'être que l'univers des faits "bruts", c'est-à-dire des faits dont on a vu qu'ils étaient en eux-mêmes arbitraires et incompréhensibles. Plusieurs chercheurs théoriques - scientifiques ou philosophes - semblent avoir pris cette décision, malgré tout, avec le résultat qu'ils ont dû insérer dans leurs théories, à la moindre occasion, ce qui est devenu une multitude d'“entités théoriques" beaucoup plus nébuleuses, que propres à vraiment améliorer notre connaissance des faits. (II est de plus en plus reconnu qu'en physique, 
par exemple, les théories contemporaines sont tout simplement stagnantes...)

Pourtant, ce que les scientifiques théoriques ne semblent pas remarquer mais que la logique de leur recherche elle-même indique, c'est qu'ils désirent atteindre au moins un des éléments qui seraient nécessaires à l'obtention d'une connaissance compréhensive. En cherchant à découvrir pourquoi les choses et leurs comportements sont comme ils sont et non pas autrement, ils cherchent une explication du déterminé (c'est-à-dire du fait que quelque chose soit déterminé). Mais parce que leurs théories explicatives ne contiennent jamais rien d'autre que des "entités» elles-mêmes déterminées (réellement ou conceptuellement), il est clair qu'elles ne sauraient leur apporter quoi que ce soit qui puisse vraiment satisfaire leur désir d'améliorer leur connaissance de l'univers déterminé. II n'en reste pas moins que la logique de leur recherche - de leur façon de procéder - continue d'exiger, pour son aboutissement concret et naturel, la découverte de réalités sans doute déterminantes (de l'univers déterminé), mais non pas elles-mêmes déterminées (à déterminer cet univers ou la connaissance qu'on en aurait). Or, la notion même des réalités déterminantes, mais non déterminées, correspond justement à la notion des réalités capables de choisir et d'agir librement, et dont les actes concrets et leurs produits arbitraires pourraient donc être connus en même temps que compris.

Les constatations précédentes trouvent une certaine confirmation dans le fait que les chercheurs théoriques éprouvent le besoin très caractéristique de simplifier et d'unifier leurs théories. S'il est bien vrai que la logique de leur recherche exige la découverte de réalités déterminantes mais non déterminées (à déterminer le déterminé), on comprend qu'ils veuillent débarrasser leur dernière théorie du plus grand nombre possible d'éléments déterminés - c'est-à-dire la simplifier - tout en visant aussi à la rendre "unitaire» ou capable d'expliquer à elle seule les faits 
les plus divers. Or, vouloir cela, c'est déjà reconnaître, plus ou moins consciemment, qu'il suffirait en principe d'un seul déterminant non déterminé (à déterminer le monde déterminé) pour qu'un monde arbitrairement déterminé devienne malgré cela connaissable et surtout compréhensible.

Si l'exposé qui précède est suffisamment juste, il implique que la recherche théorique n'est qu'un jeu ou un défi à relever, sans doute agréable pour plusieurs, mais dépourvu de «sens». Pour qu'il puisse acquérir un sens, il faudrait que cette recherche soit poursuivie non seulement pour l'agrément, ou par souci de gagner sa propre vie, mais aussi par intérêt pour des personnes. Et qu'elle soit une expression concrète de cet intérêt. On voit assez bien comment la pratique d'un sport, des arts dramatiques, plastiques ou musicaux, puisse être l'expression active d'un intérêt pour les personnes, du moins pour celles que de telles performances réussissent ou bien à distraire d'un quotidien parfois morne ou pénible ou bien à faire vivre des expériences humainement stimulantes et encourageantes. On voit très bien, aussi, comment la recherche en sciences pratiques et techniques peut favoriser le bienêtre de beaucoup de gens et être poursuivie par intérêt pour ces personnes. Mais on voit moins bien, par contre, comment les chercheurs purement théoriques - ceux qui voudraient connaitre le monde uniquement pour le connaître - pourraient effectuer leurs recherches par intérêt pour des personnes, surtout lorsqu'ils ne semblent pas faire de découvertes intéressantes pour les humains, qu'ils ne cherchent même pas à en faire bénéficier ceux-ci (et pour cause!) et que plusieurs d'entre eux ne peuvent pratiquer leur sport théorique qu'au moyen d'un salaire et de subventions arrachés à leurs concitoyens.

Quel serait donc le sens de la recherche théorique, de cette recherche qui ne réussit jamais qu'à avorter ou à stagner? S'il devait être découvert un jour, il semble que ce serait par la découverte du sens de notre univers de faits déterminés et arbitraires et par la découverte, en 
même temps, du sens de la vie humaine, si souvent déterminée, factuelle, et arbitraire elle aussi. À cause de la sensibilité d'aujourd'hui en ce qui a trait aux droits des personnes de même qu'au respect et à l'intérêt qui seraient dus à chacune de celles-ci, on peut penser qu'il y a lieu d'espérer, qu'un nombre croissant de personnes voudront donner un sens social à leurs occupations, y compris les chercheurs qui désirent réellement connaître l'univers, c'est-à-dire en comprendre, enfin, le «sens». Et peut-être même l'exprimer.

Gilles Lane

Département de philosophie Université de Montréal 